\title{
Influence of Parameters of a Printing Plate on Photoluminescence of Nanophotonic Printed Elements of Novel Packaging
}

\author{
Olha Sarapulova and Valentyn Sherstiuk \\ Publishing and Printing Institute, National Technical University of Ukraine "Kyiv Polytechnic Institute", 1/37 Yangel Street, \\ Kyiv 03056, Ukraine \\ Correspondence should be addressed to Olha Sarapulova; olhasarapulova@gmail.com
}

Received 21 June 2015; Revised 12 August 2015; Accepted 17 August 2015

Academic Editor: Carlos R. Cabrera

Copyright (C) 2015 O. Sarapulova and V. Sherstiuk. This is an open access article distributed under the Creative Commons Attribution License, which permits unrestricted use, distribution, and reproduction in any medium, provided the original work is properly cited.

\begin{abstract}
In order to produce nanophotonic elements for smart packaging, we investigated the influence of the parameters of screen and offset gravure printing plates on features of printed application of coatings with nanophotonic components and on parameters of their photoluminescence. To determine the dependence of luminescence intensity on the thickness of solid coating, we carried out the formation of nanophotonic solid surfaces by means of screen printing with different layer thickness on polypropylene film. The obtained analytical dependencies were used to confirm the explanation of the processes that occur during the fabrication of nanophotonic coverings with offset gravure printing plates. As a result of experimental studies, it was determined that the different character of the dependency of total luminescence intensity of nanophotonic elements from the percentage of a pad is explained by the use of different types of offset gravure printing plates, where the size of raster points remains constant in one case and changes in the other case, while the depth of the printing elements accordingly changes or remains constant. To obtain nanophotonic areas with predetermined photoluminescent properties, the influence of investigated factors on changes of photoluminescent properties of nanophotonic printed surfaces should be taken into consideration.
\end{abstract}

\section{Introduction and Background}

Printed nanophotonic elements, which contain nanoscale photoactive components with fluorescent properties, are designed to protect printing products against counterfeiting and extend functionality of smart food packaging. Such elements of smart packaging react to changes that occur in the internal or external environment of food packaging. If the product is stored in inappropriate conditions and/or spoilage products occur in a packaged product (breakdown products of proteins, organic acids, alcohols, carbon dioxide, etc.), printed nanophotonic elements on the outer or inner surface of a packaging influenced by such conditions over time and/or in contact with the mentioned substances change their properties. The change of the properties can be recorded instrumentally (if nanophotonic element is part of an electronic or photonic sensor) or visually. In the latter case nanophotonic elements of smart packaging alter the colour or intensity of luminescence and thus inform a consumer about the suitability of a packaged product for consumption.

Printing production of nanophotonic elements of modern packaging on various surfaces is complicated by the necessity to consider the impact of process parameters on nanophotonic components of compositions that are applied to the surface. One of the most important groups of parameters [1] are the characteristics of a printing plate. Factors such as screen grid parameters for screen printing, and depth of printing elements and screen frequency for gravure printing plates (including offset gravure printing plates), determine the thickness of the printing ink layer and the resulting cover layer, which can influence the fluorescent properties of 
coatings obtained [2]. Thus, to obtain nanophotonic elements of novel packaging with predetermined photoluminescence properties (intensity, colour, and subsequent changes of parameters in the process of functioning), technological parameters must be considered and if necessary the adjustments should be made in the process of manufacturing of printing plates.

In the literature there is little data on research on the influence of parameters of a printing plate on the process of application of photonic and nanophotonic coatings. There is extensive research on the use of different nanoparticles in printed smart packaging $[3,4]$. There are general studies of application of printed layers containing nanoparticles for food packaging $[5,6]$. The use of $\mathrm{ZnO}$ nanoparticles has several advantages over the use of other luminescent compounds as nano- $\mathrm{ZnO}$ is primarily safe, possesses antimicrobial properties, absorbs UV radiation $[3,6]$, and changes the intensity of luminescence in contact with decomposition products of organic matter due to destruction of nano- $\mathrm{ZnO}$ crystals and the formation of aggregates without luminescent properties [6]. Informing consumers may be carried out by changing certain properties of packaging elements such as optical, electrical, and geometrical properties [4]. There are studies of the influence of parameters of nanoscale coating photoactive elements without reference to the parameters of a printing plate $[1,2]$. In case of screen printing plate it is important to select the grid, and in case of offset gravure printing it is important to choose a method of manufacture (laser engraving on metal plates or production of photopolymer plates), because these factors determine the parameters of the printing plate that significantly affect photoluminescence of received printed coatings. Thus there is need for researching the influence of parameters of a printing plate on photoluminescent characteristics of nanophotonic printed coatings in order to obtain effective nanophotonic elements to protect printing products against counterfeiting and/or manufacture of smart packaging.

The aim of the work is to study the influence of technological parameters (related to a printing plate) on the process of applying printed nanophotonic coatings and their photoluminescent properties to determine the optimal parameters of a printing plate for the highest quality printing reproduction of nanophotonic elements of novel packaging with predetermined photoluminescent parameters.

\section{Materials and Methods}

2.1. Materials. Nanophotonic composition based on colloidal solution of zinc oxide ( $\mathrm{ZnO})$ nanocrystals sized $4.5-6 \mathrm{~nm}$ in doubly distilled ethanol [7] and polyvinylpyrrolidone (PVP) with molecular mass of 360000, which is designed for use in the manufacture of printing nanophotonic coatings [8], was applied to polypropylene film by screen and offset gravure printing.

2.2. Methods. The investigated parameters of a printing plate are the following: screen grid frequency and thickness for screen printing and depth of printing elements and screen frequency for gravure printing plates (including offset

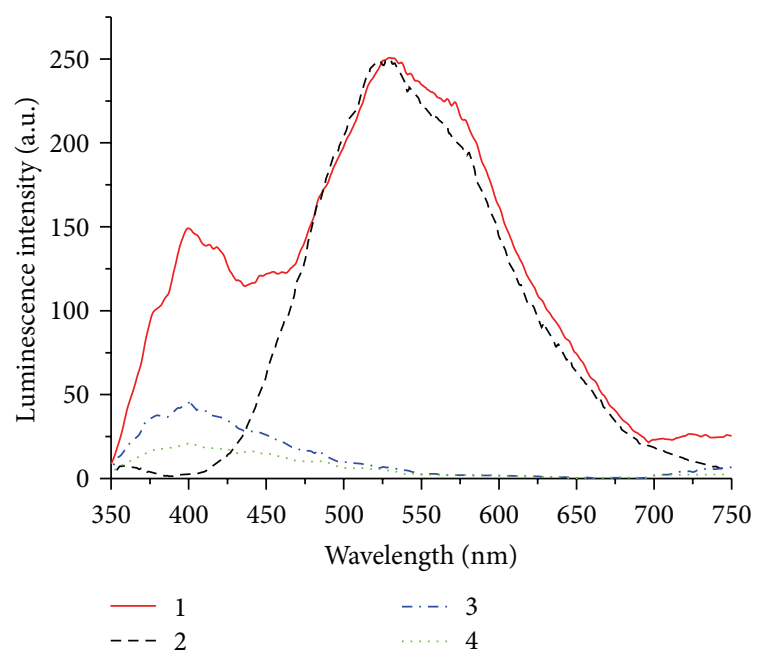

FIGURE 1: The luminescence spectra of 1: nanophotonic layer (PVP and $\mathrm{ZnO}$ ) on polypropylene film (layer thickness is $100 \mu \mathrm{m}$ ); 2: colloidal solution of $\mathrm{ZnO}$ nanocrystals, $[\mathrm{ZnO}]=2 \cdot 10^{-2} \mathrm{~mol} / \mathrm{L}$; 3: layer of PVP without $\mathrm{ZnO}$ on polypropylene film; and 4: polypropylene film.

gravure printing plates). The investigated parameters of photoluminescence of the obtained printed layers are intensity and colour or photoluminescence. The colour is determined by the wavelength of the peaks of photoluminescence spectra.

To obtain printed solid areas of different thickness, screen printing plates with different mesh was used and the number of layers varied. To obtain printed raster areas of different thickness with different screen frequency, metal gravure printing plates were used, and diameter of raster points was varied, while screen frequency was constant $(50$ lines $/ \mathrm{cm})$ to obtain the raster areas of $12.5 \%, 25 \%, 37.5 \%, 50 \%, 67.5 \%$, and $87.5 \%$ with constant thickness of the coating layer. Photopolymer gravure printing plates were also used; the thickness of the coating layer varied with changing the depth of printing elements $(10-100 \mu \mathrm{m})$ at constant screen frequency (50 lines/cm) to obtain $100 \%$ areas. Pad printing was used to simulate offset gravure printing due to reasons of technological similarity. Photoluminescence spectra were obtained with fluorescence spectrometer Perkin Elmer LS 55.

\section{Results and Discussion}

Nanophotonic element in printed layers on polypropylene film has photoluminescence spectrum with two peaks-at $\lambda=$ $400 \mathrm{~nm}$ and $\lambda=525 \mathrm{~nm}$ (Figure 1, curve 1); thickness of layer is $100 \mu \mathrm{m}$. The peak at $\lambda=525 \mathrm{~nm}$ is caused by the colloidal solution of $\mathrm{ZnO}$ nanocrystals in nanophotonic composition (Figure 1, curve 2). The peak at $\lambda=400 \mathrm{~nm}$ is caused by PVP as part of nanophotonic composition and polypropylene film as a substrate. Despite the fact that the PVP luminescence (Figure 1, curve 3) and polypropylene film luminescence (Figure 1, curve 4) are minor, due to energy transfer the resulting nanophotonic composition has a pronounced peak of luminescence at $\lambda=400 \mathrm{~nm}$. The difference in position of this peak relative to the position of the peak at $\lambda=525 \mathrm{~nm}$ 


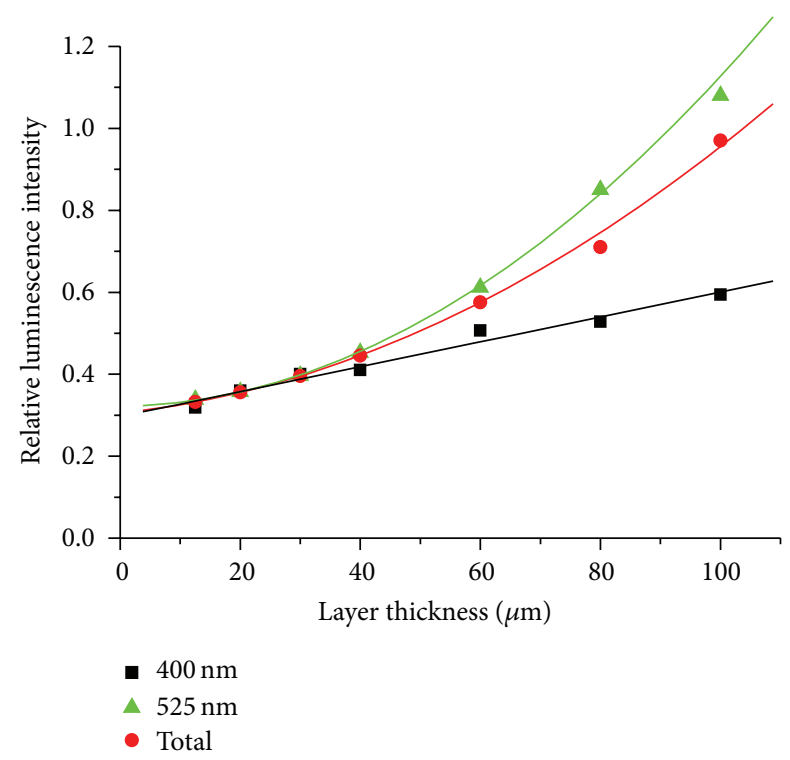

FIGURE 2: The dependencies of the luminescence intensity on the thickness of solid layers.

affects the colour of the luminescence of the coating, as well as the total intensity of its luminescence.

3.1. The Influence of Layer Thickness of a Solid Ink Layer on Its Luminescence Intensity. To determine the influence of layer thickness of a solid ink layer on its luminescence intensity the layers were deposited onto polypropylene film using screen printing with different layer thickness. Figure 2 shows the change in luminescence intensity for peaks at $\lambda=400 \mathrm{~nm}$ and $\lambda=525 \mathrm{~nm}$ and total luminescence intensity.

From the resulting discrete experimental data, analytical dependencies were obtained based on polynomial curves. The analytical dependencies allow determining the thickness $\delta$ and predicted luminescence intensity $I_{400}$ of the peak at $\lambda=$ $400 \mathrm{~nm}$ of a nanophotonic layer:

$$
I_{400}=0.0032 \delta+0.3003
$$

luminescence intensity $I_{525}$ of the peak at $\lambda=525 \mathrm{~nm}$ :

$$
I_{500}=0.00008 \delta^{2}-0.00003 \delta+0.3259
$$

and total luminescence intensity $I$ :

$$
I=0.00005 \delta^{2}+0.0015 \delta+0.3055 .
$$

These dependencies were used to predict the intensity of luminescence of nanophotonic layers obtained using screen printing and to explain deviations of dependencies.

\subsection{The Influence of Layer Thickness of a Raster Ink Layer on Its} Luminescence Intensity. For layers obtained with pad (offset gravure) printing, photopolymer printing plates were used, where raster areas of $25 \%, 50 \%, 75 \%$, and $100 \%$ had constant raster dot size, while depth of printing elements varied and thus the thickness of the coating layer varied. Experimental

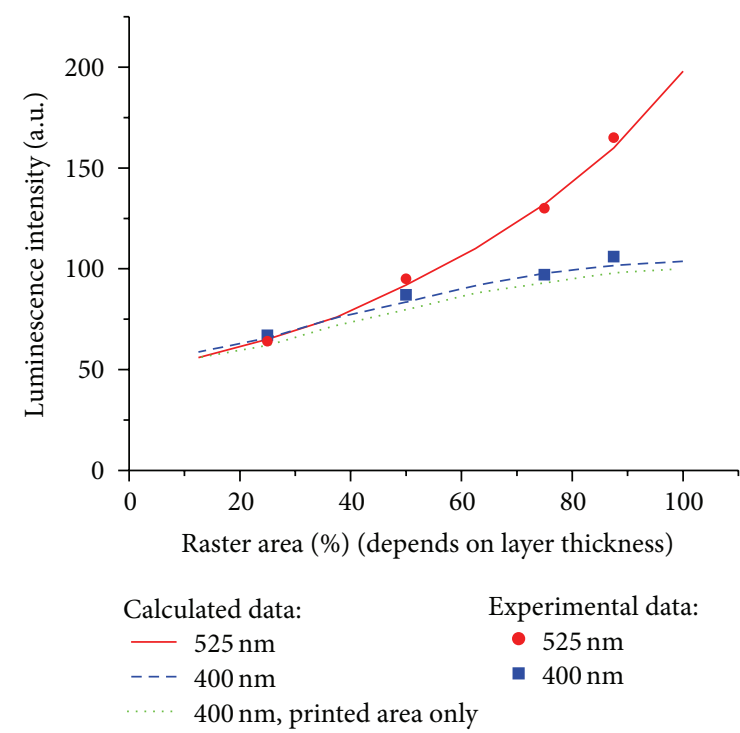

FIgURE 3: The dependencies of the luminescence intensity of nanophotonic elements on the layer thickness, which is regulated by the variable depth of printing elements.

dependencies of the luminescence intensity of nanophotonic elements on the layer thickness were obtained and compared with the calculated data (Figure 3).

Calculated data were calculated using (1) and (2) for the peak of luminescence at $\lambda=400 \mathrm{~nm}$ and $\lambda=525 \mathrm{~nm}$ in accordance with the layer thickness of $15-100 \mu \mathrm{m}$, and luminescence unprinted areas of polymer substrate $(12.5 \%$ of the total printed area) were taken into account. As shown in Figure 3, experimental data are consistent with the calculated values of the intensity of luminescence.

For layers obtained with pad printing metal printing plates were also used, manufactured by laser engraving, where raster areas of $12.5 \%, 25 \%, 37.5 \%, 50 \%, 67.5 \%$, and $87.5 \%$ had constant depth of printing elements (layer thickness $80 \mu \mathrm{m}$ ), while raster dot size varied and thus the printed area of the coating layer varied. Experimental dependencies of the luminescence intensity of nanophotonic elements on the percentage of raster areas were obtained and compared with the calculated data (Figure 4).

The data were calculated using analytical dependencies (1) and (2) for peaks at $\lambda=400 \mathrm{~nm}$ and $\lambda=525 \mathrm{~nm}$ in accordance with the thickness of the coating layer of $80 \mu \mathrm{m}$, and the luminescence of unprinted areas of the polymer substrate was taken into account, which varied from $87.5 \%$ to $12.5 \%$ for $12.5 \%$ to $87.5 \%$ of the raster areas, respectively. As shown in Figure 4, experimental data are consistent with the calculated values of the intensity of luminescence.

3.3. The Influence of Raster Area Percentage of Ink Layer on Its Total Luminescence Intensity and Colour. The influence of raster area percentage on total luminescence intensity of printed nanophotonic elements obtained with two different types of pad (offset gravure) printing plates was calculated using the analytical dependence (3) and taking into account the luminescence of unprinted areas of polymer substrates. 


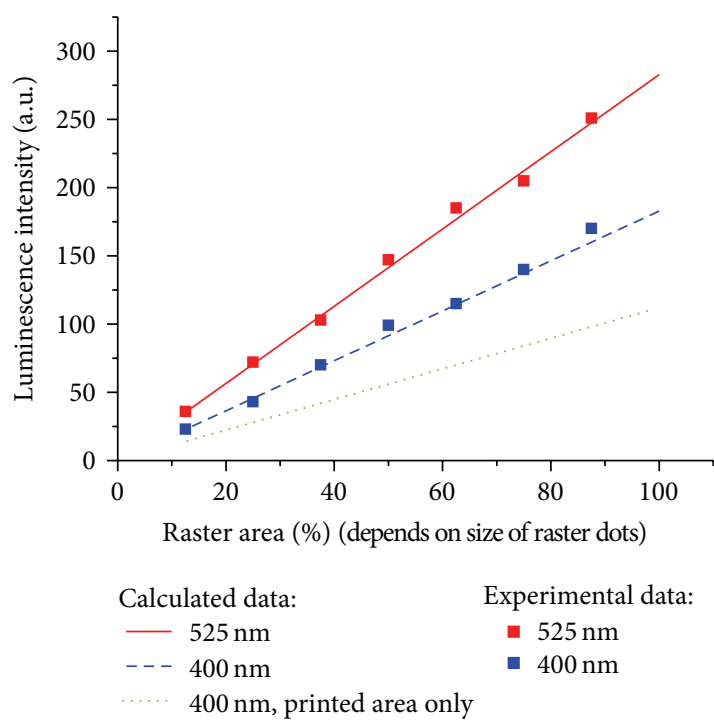

FIgURE 4: The dependence of the luminescence intensity of nanophotonic elements on continuity of coverage of printed areas, which is regulated by the percentage of raster areas of pad printing impressions with variable dot size and constant depth of printing elements of a printing plate.

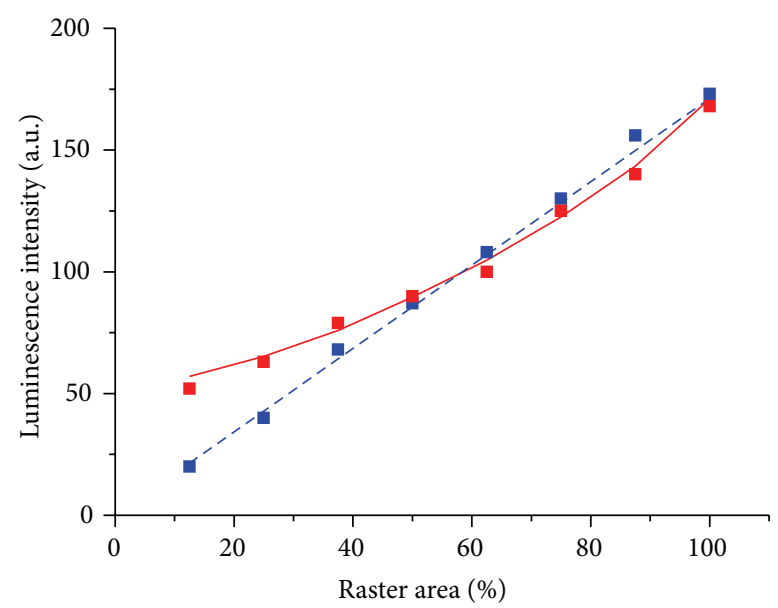

Calculated data:

— For variable layer thickness (photopolymer printing plates)

- - For variable raster dot size (metal printing plate)

Experimental data:

For variable layer thickness (photopolymer printing plates)

For variable raster dot size (metal printing plate)

FIGURE 5: The dependence of total luminescence intensity of nanophotonic elements on percentage of raster areas obtained with two different types of pad (offset gravure) printing plates.

As can be seen in Figure 5, the experimental data are consistent with the calculated values of the intensity of luminescence.

Thus, different shape of dependencies of the total luminescence intensity of nanophotonic elements on percentage of raster areas can be explained as follows. When photopolymer pad printing plates are used, raster dots size (i.e., printed and unprinted surface area) remains constant, determining luminescence at $\lambda=400 \mathrm{~nm}$ caused by the luminescence of polypropylene substrate in unprinted areas and luminescence of PVP in printed areas. Luminescence at $\lambda=525 \mathrm{~nm}$ is caused by luminescence of nano- $\mathrm{ZnO}$ as a component of nanophotonic composition, obviously in printed areas. The variable depth of printing elements (i.e., layer thickness) defines a parabolic dependence of the luminescence intensity at $\lambda=400 \mathrm{~nm}$ on layer thickness of printed areas of nanophotonic elements $(30-120 \mu \mathrm{m})$ caused by the luminescence of a polymer (PVP) as a component of the nanophotonic composition. The variable depth of printing elements also defines exponential dependence of the luminescence intensity on the layer thickness of printed areas of nanophotonic elements $(30-120 \mu \mathrm{m})$ caused by the luminescence of $\mathrm{ZnO}$ as a component of the nanophotonic composition.

The peak at $\lambda=525 \mathrm{~nm}$ is always higher than the peak at $\lambda$ $=400 \mathrm{~nm}$ due to relatively small unprinted area of raster areas and thus insignificant contribution of the own luminescence of polypropylene film into the peak size at $\lambda=400 \mathrm{~nm}$. It means the luminescence colour will have yellow shades.

When using metal pad printing plates, the raster dot size (i.e., printed area) varies, determining the linear dependence of the luminescence intensity at $\lambda=400 \mathrm{~nm}$ on the area of unprinted areas caused by the luminescence of the polymer (PVP). It also determines the linear dependence of the luminescence intensity at $\lambda=400 \mathrm{~nm}$ and $\lambda=525 \mathrm{~nm}$ on the printed area of nanophotonic elements (12.5-87.5\% of $12.5-$ $87.5 \%$ raster printed areas, resp.), caused by the luminescence of PVP and nano- $\mathrm{ZnO}$ as components of the nanophotonic composition. The depth of printing elements of a printing plate remains constant (i.e., layer thickness), determining the luminescence at $\lambda=400 \mathrm{~nm}$ and $\lambda=525 \mathrm{~nm}$ of printed areas of nanophotonic elements (layer thickness is $80 \mu \mathrm{m}$ ), caused by the luminescence of PVP and $\mathrm{ZnO}$ as components of the nanophotonic composition.

The peak at $\lambda=400 \mathrm{~nm}$ can be higher than the peak at $\lambda=525 \mathrm{~nm}$ due to a larger unprinted areas with the same percentage as compared to the areas obtained with photopolymer printing plates and a significant contribution of the own luminescence of polypropylene film to the peak at $\lambda=400 \mathrm{~nm}$. Thus the luminescence colour can have yellow shades (when the peak at $\lambda=525 \mathrm{~nm}$ is higher than the peak at $\lambda=400 \mathrm{~nm}$ ) and green shades (when the peak at $\lambda=400 \mathrm{~nm}$ is higher than the peak at $\lambda=400 \mathrm{~nm})$.

3.4. Research Summary Data. It was determined that the thickness of ink layer determines the basic parameter of photoluminescence of a nanophotonic printed element-its intensity. Other parameters allow less extreme changes to photoluminescence intensity and moderate changes to colour of photoluminescence.

The optimum values of the parameters of a printing plate should be chosen according to which colour and luminescent intensity are to be obtained. Therefore, for every designed nanophotonic element the obtained dependencies should be used to determine the needed parameters of a printing plate. For this purpose, the research summary data are presented in Table 1. 
TABLE 1: Influence of parameters pad (offset gravure) printing plates on the luminescence characteristics of printed nanophotonic elements.

Constant
Parameter
(i) The nature of the influence on
the luminescence peak at $\lambda=$
$400 \mathrm{~nm}$
$400 \mathrm{~nm}$

(ii) The nature of the influence on the luminescence peak at $\lambda=$ $525 \mathrm{~nm}$

Variable Parameter
(i) Nature of the influence on the
luminescence peak at $\lambda=400 \mathrm{~nm}$

(ii) Nature of the influence on the luminescence peak at $\lambda=525 \mathrm{~nm}$

\section{Conclusions}

As a result of experimental research the deposition of nanophotonic composition based on nanosized $\mathrm{ZnO}$ and polyvinylpyrrolidone was carried out by the means of screen and pad (offset gravure) printing onto polypropylene in order to obtain printed nanophotonic elements. These elements are intended for smart packaging and security printing, including printing protecting products against forgery. The influence of parameters of printing plate on photoluminescent properties of obtained coatings was investigated. It was determined that the parameters of pad (offset gravure) printing plates have different impact on luminescence of nanophotonic elements depending on the type of their constant and variable parameters - whether the percentage of

Type of the dependence of total

luminescence intensity on
Depth of printing elements on a printing plate (i.e., engraving depth, layer thickness)

Constant parameter specifies the luminescence of printed areas (layer thickness is $80 \mu \mathrm{m}$ ), accounted to the luminescence of the polymer (PVP) as a component of the nanophotonic composition component of the nanophotonic

Constant parameter specifies the luminescence of printed areas (87,5\% of the total raster area), accounted to the luminescence of nano- $\mathrm{ZnO}$ as a component Depth of printing elements on a printing plate (i.e., engraving depth, layer thickness)

Constant parameter specifies the luminescence of printed areas (layer thickness is $80 \mu \mathrm{m}$ ), accounted to the luminescence of nano- $\mathrm{ZnO}$ as a component of the nanophotonic composition

Size of raster dots

(i.e., printed and unprinted surface area)

Variable parameter specifies the linear dependence of the luminescence intensity on the unprinted area of the polymer substrate (of $12,5-87,5 \%$ raster printed areas, resp.), accounted to the luminescence of PVP and-for printed areas-nano- $\mathrm{ZnO}$ as components of the nanophotonic composition

Variable parameter specifies the linear dependence of the luminescence intensity on the unprinted area of the polymer substrate (of $12,5-87,5 \%$ raster printed areas, resp.), accounted to the luminescence of nano- $\mathrm{ZnO}$ as a component of the nanophotonic composition

Linear

For more luminescence intensity spot at $55 \%$; for luminescence spectrum with a larger peak at $\lambda=400 \mathrm{~nm}$ (green shades of luminescence colour)

For more luminescence intensity spot at $55 \%$; for luminescence spectrum with a larger peak at $\lambda=525 \mathrm{~nm}$ (yellowish shades of luminescence colour)

pads is regulated by the size of raster dots of the thickness of printed layers. It is caused by the fact that in the first case at various percentages of raster area raster dots have the same size; that is, printed and unprinted surface area is constant, while the depth of printing elements on a printing plate varies; that is, the thickness of a printed layer varies; in the second case it is vice versa. These factors cause different type of changes of photoluminescent properties of nanophotonic printed coatings, which should be considered in order to obtain nanophotonic elements of smart packaging with predetermined photoluminescent properties. Further research is needed to determine the influence of parameters of printing and postprinting processes in order to ensure the proper functionality of the nanophotonic elements of novel packaging. 


\section{Conflict of Interests}

The authors declare that there is no conflict of interests regarding the publication of this paper.

\section{Acknowledgment}

The studies were conducted with the support of the Ministry of Education and Science of Ukraine (Grant 2873 p).

\section{References}

[1] O. O. Sarapulova and V. P. Sherstiuk, "Problems of printing production of new nanoscale packaging with photoactive elements," Technology and Technique of Typography, vol. 2, pp. 4657, 2013.

[2] O. O. Sarapulova and V. P. Sherstiuk, "Technological features of application of nanophotonic elements of packaging by screen printing," Technology and Technique of Typography, vol. 3, no. 41, pp. 18-26, 2013.

[3] X. H. Li, Y. G. Xing, W. L. Li, Y. H. Jiang, and Y. L. Ding, "Antibacterial and physical properties of poly(vinyl chloride)based film coated with $\mathrm{ZnO}$ nanoparticles," Food Science and Technology International, vol. 16, no. 3, pp. 225-232, 2010.

[4] K. L. Yam, P. Takhistov, and J. Miltz, "Intelligent packaging: concepts and applications," Journal of Food Science, vol. 70, no. 1, p. 10, 2005.

[5] P. Suppakul, J. Miltz, K. Sonneveld, and S. W. Bigger, "Active packaging technologies with an emphasis on antimicrobial packaging and its applications," Journal of Food Science, vol. 68, no. 2, pp. 408-420, 2003.

[6] A. Emamifar, M. Kadivar, M. Shahedi, and S. SoleimanianZad, "Preparation and evaluation of nanocomposite LDPE films containing Ag and $\mathrm{ZnO}$ for food-packaging applications," Advanced Materials Research, vol. 129, pp. 1228-1232, 2010.

[7] V. V. Shvalagin, A. L. Stroyuk, and S. Y. Kuchmii, "Role of quantum-sized effects on the cathodic photocorrosion of $\mathrm{ZnO}$ nanoparticles in ethanol," Theoretical and Experimental Chemistry, vol. 40, no. 6, pp. 378-382, 2004.

[8] V. P. Sherstiuk, V. V. Shvalagin, O. O. Sarapulova, and V. M. Granchak, "Luminescent films based on nanoscale zinc oxide and polyvynylpyrrolydone and their functional characteristics," in Proceedings of the 6th International Scientific Conference 'Functional Base of Nanoelectronics', pp. 250-253, KNURE, Alushta, Ukraine, October 2013. 

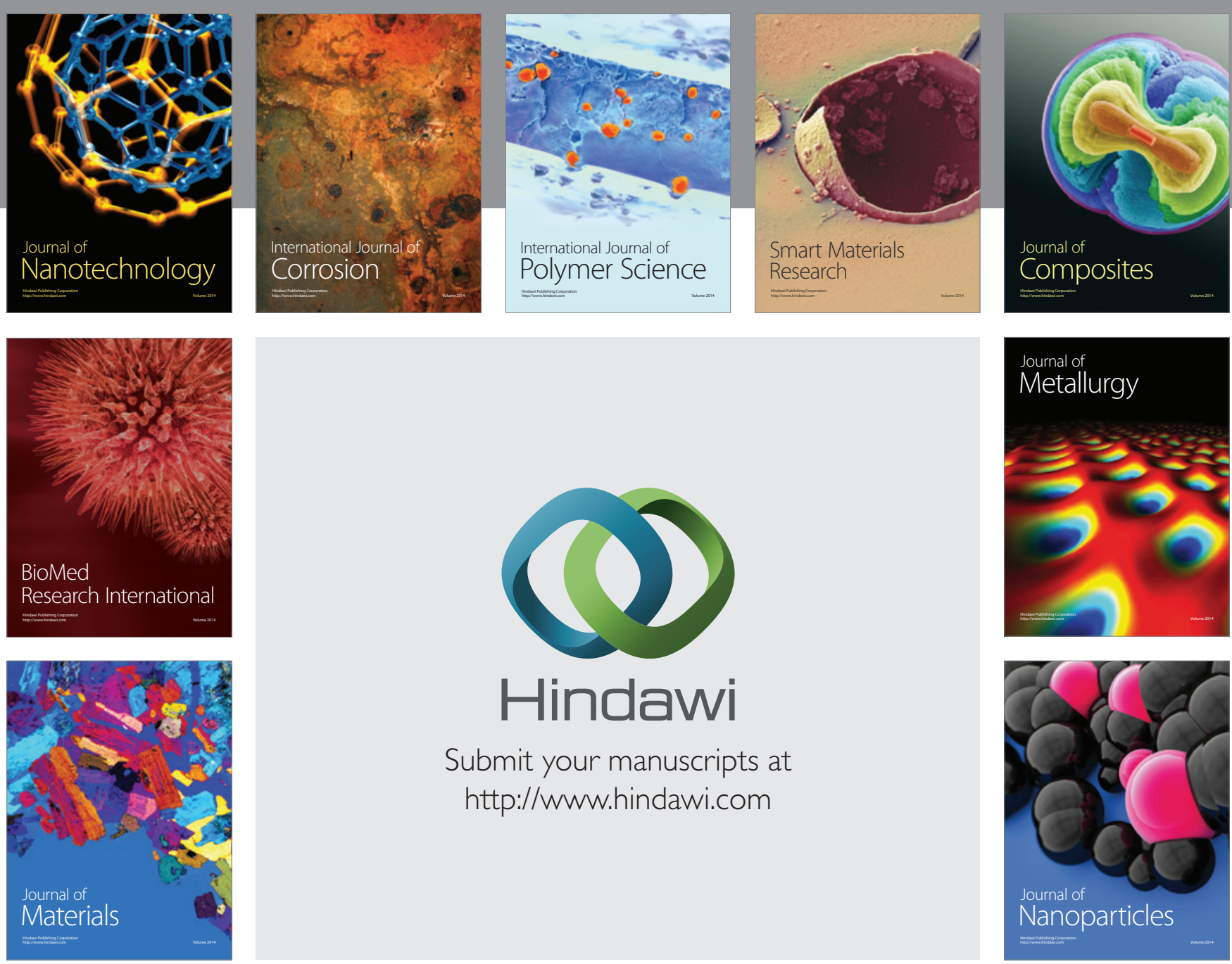

Submit your manuscripts at http://www.hindawi.com
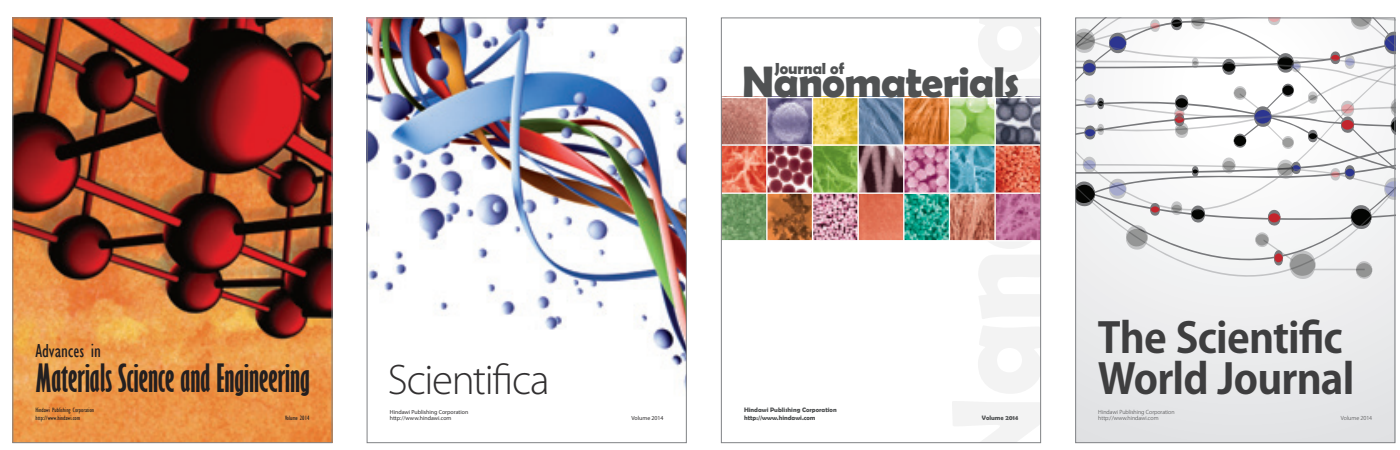

\section{The Scientific World Journal}
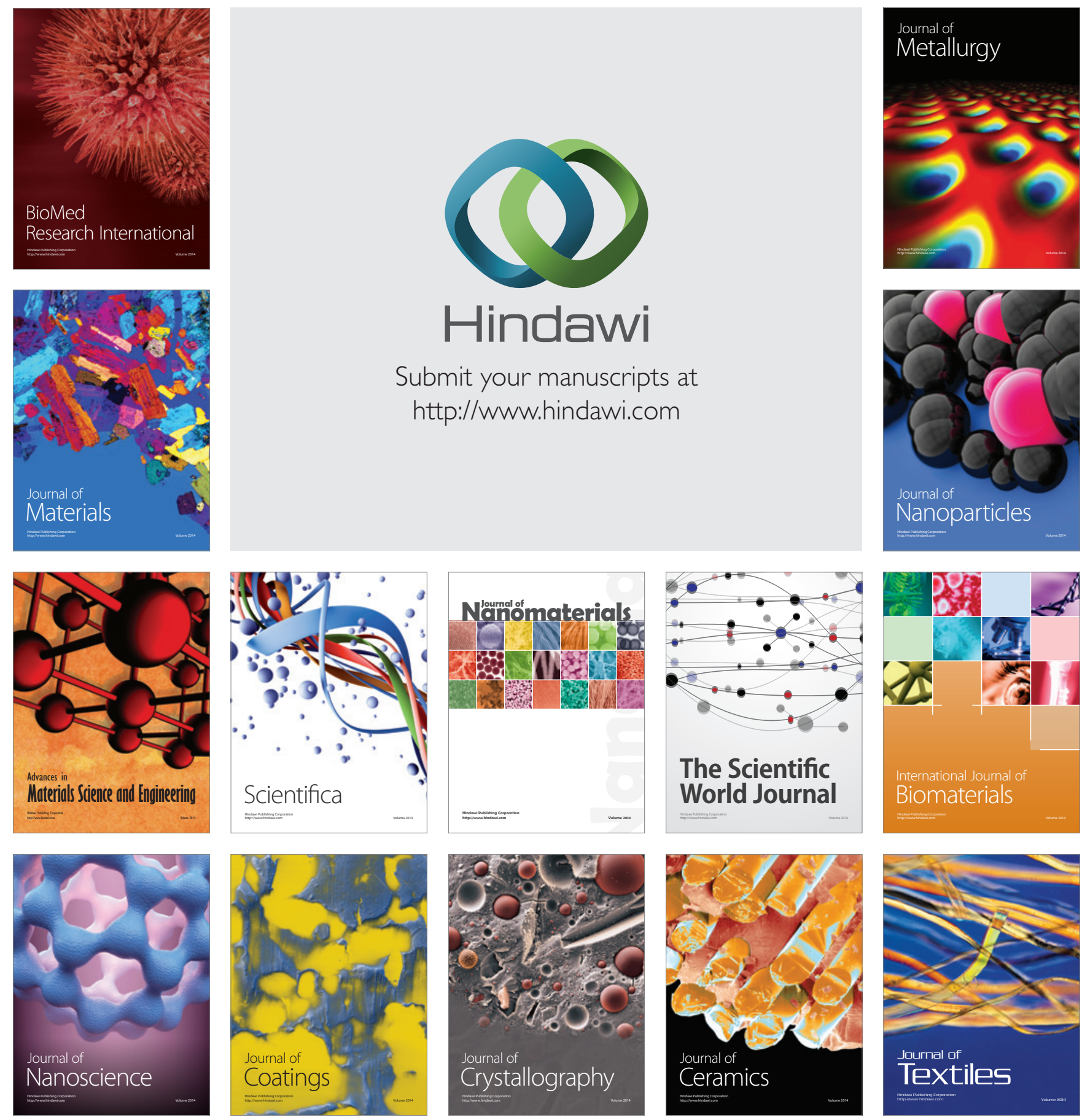